\title{
Whole-electronic line-focus light-scanner for active thermography
}

\author{
by Jun Hoshimiya and Tsutomu Hoshimiya
}

\author{
* Tohoku Gakuin Univ., Chuo-1-13-1, Tagajyo, 985-8537, Japan, tpth@tjcc.tohoku-gakuin.ac.jp
}

\begin{abstract}
In this paper, a whole-electronic line-focus light-scanning apparatus for active thermography, which can be fabricated as a mobile-size, was proposed, designed and fabricated. A multiple column LED/LD array was coupled with a focusing lens to construct scanning optics with variable magnification. Each LED/LD stack was selected and lighted with a multi-channel analog output device controlled with a PC. The light power, scanning speed, individual irradiation time duration and other scanning parameters of LED/LD array were easily adjusted with a control software LabVIEW.
\end{abstract}

\section{Introduction}

Active-thermography with a line-focus heat source, or line scan thermography (LST) has been developed due to its rapid inspecting ability of large areas with minimum time. Recently mechanically-scanned LSTs with both optical and electromagnetic heating scheme have been developed as non-destructive evaluation (NDE) tools [1-3] and even sold as a product [4].

A mechanical scanning system has the advantage of high-precision, however it has disadvantage of heavy weight and high price. A galvano-scan system improved the disadvantage of the former type, however it has also the limitation to achieve a handy-sized apparatus.

For applications of moving spot thermography $[5,6]$, the importance of the adjustment of the scanning speed to thermal diffusivity of the specimens is described. Similarly in line-scanning scheme, the adjustment of scanning speed is also important. As shown in the Appendix, specimens with larger thermal diffusivity such as brass or aluminum make the NDE of inner defects difficult so that they require fast scanning of the beam. Therefore, easy adjustment of scanning speed is essential in scanning active thermography with both point and line scanning schemes.

In this paper, a whole-electronic light scanning apparatus for point- and line-focus system with LED/LD arrays, which can be fabricated as a mobile-size, was proposed for the first time. Furthermore, a whole-electronic scanner for LST was specially designed and fabricated. Due to the low-price character of high-power LEDs and LDs (\$1-\$3.5 or 0.72.5 Euro/piece), the present apparatus realized quasi-continuous scanning of the line heat-source over the specimen surface. The control hardware and software are easily designed with the conventional electronics technology using the software LabVIEW( ${ }^{\mathrm{TM}}$ National Instruments). (This apparatus can be realized only by the hardware technique such as a digital demultiplexer.)

The heat source power and beam scanning speed matching with respect to the thermal properties of the specimens can be easily adjusted with a software at the real-time on the PC screen.

\section{Principle and experimental apparatus}

\subsection{Principle}

The principle of scanning and illumination of point- or line-focus light beam [7] was schematically shown in Fig. 1. The $N$-channel LED/LD array is sequentially fired with a period and duration of $T$ and $\tau$ respectively. Furthermore, the LED/LD array-lens distance and lens-specimen distance are assumed to be $a$ and $b$ respectively, the magnification of the image $m$ of the lens with the focal length $f$ is calculated to be

by the image formation formula of the lens.

$$
\mathrm{m}=\frac{b}{a} \quad \frac{1}{a}+\frac{1}{b}=\frac{1}{f}
$$

If $\mathrm{N}$-channels of LED/LD array were used and the total length of scanning is $\mathrm{L}$, then the scanning speed $v$ is determined by the switching time difference $\mathrm{T}$ by the relation

$$
\mathrm{v}=\frac{L}{N T} \text {. }
$$

Other parameters, the illumination light power $(P)$ and illumination time $(\tau)$ can be adjusted by changing analog output voltage $(P)$, pulse width $(\tau)$, and pulse period $(T)$. The scanning distance $L$ can be adjustable by the magnification of lens imaging system $m$ given by the equation (1).

\subsection{Apparatus}


The experimental setup is shown in Fig. 2. For a single element of an optical source array, a white LED (maximum luminous flux of $200 \mathrm{~lm}$ ) or an infrared LD (maximum power $100 \mathrm{~mW}$ ) was selected as candidates. A thermal image was observed by a thermo-tracer (NEC San-ei, TH 9100) with a temperature resolution of 0.06K. The real-time (30 flames/sec) thermal response was recorded as a movie by a PC connected with an IEEE 1394 bus.

Four to six LEDs were set as an individual column of 16-columned LED array. Each LED column was sequentially selected and lighted with a power transistor switching/drive circuit which was controlled by one of a 16 channel analog output device (cDAQ+NI9264) controlled with a software LabVIEW. Each channel emitted LED light was coupled with a focusing lens (spherical or cylindrical) after the traverse through the metal light guide. The 16 channel optical line sources were imaged with a reduced projection system as 16 line heat sources.

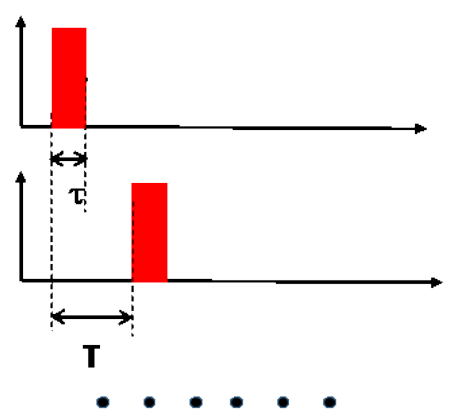

a

b
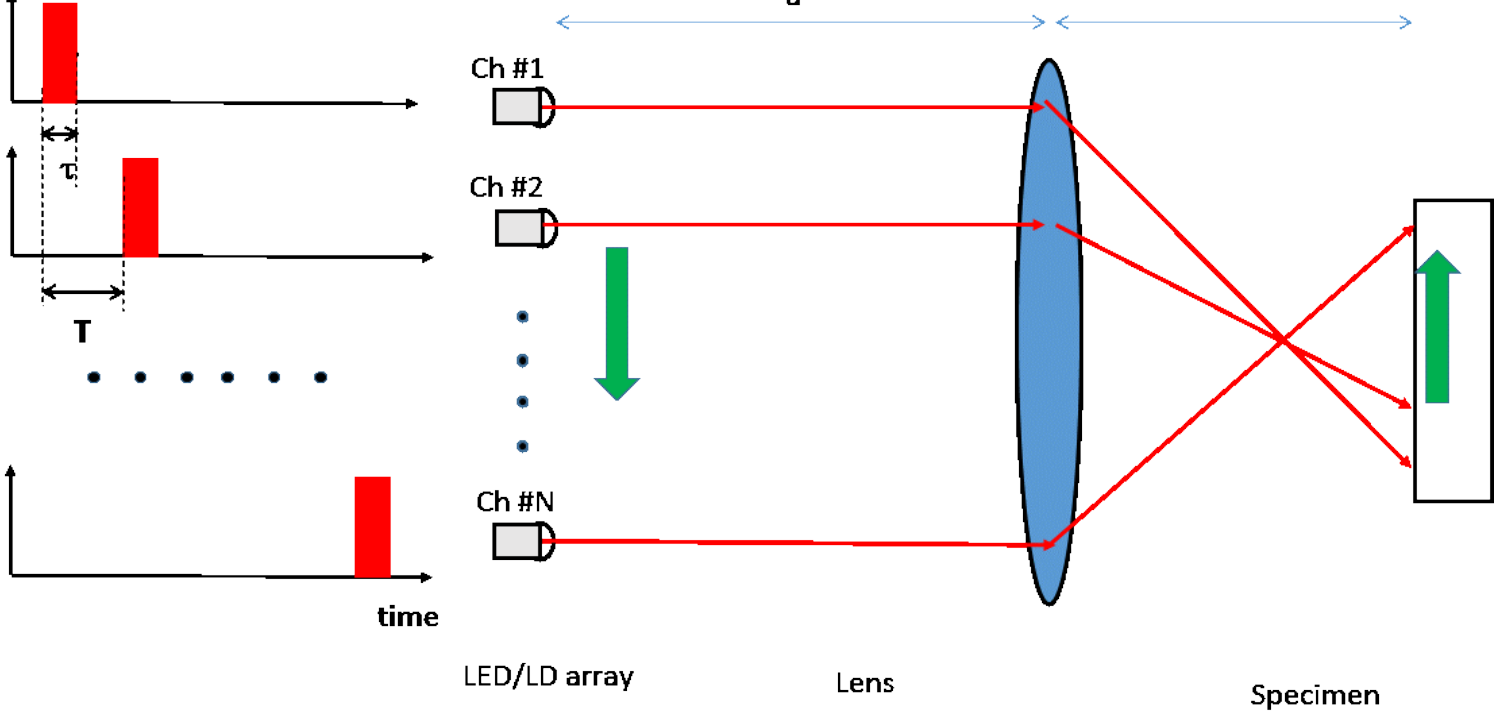

Fig. 1 Principle of electronic scanning scheme

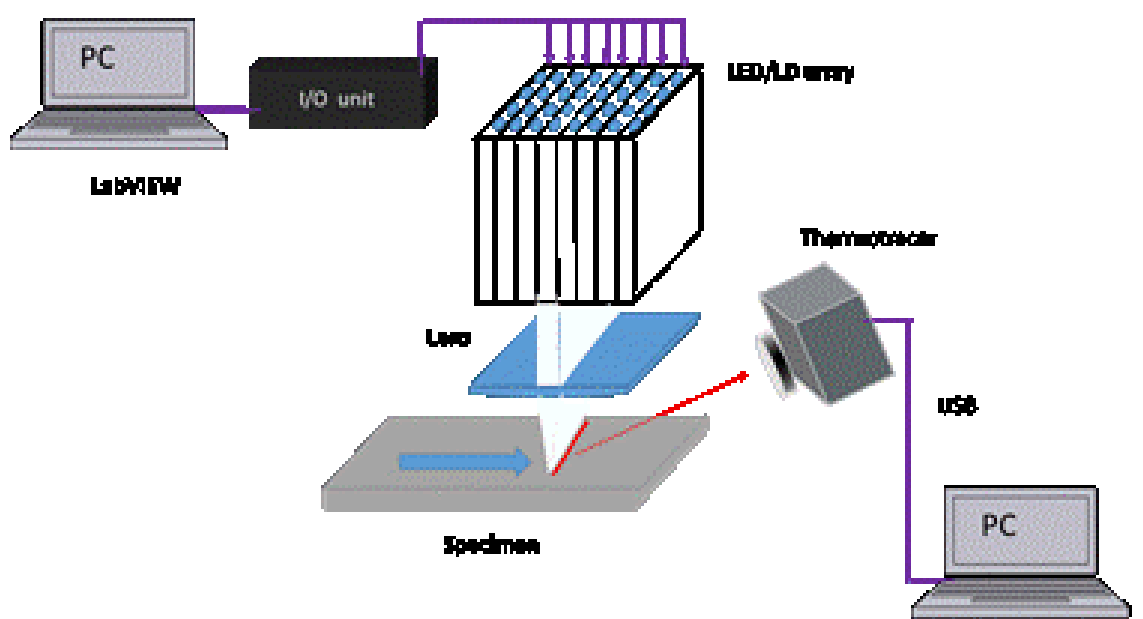

Fig. 2 Basic experimental setup

\subsection{Software}

Control of beam scanning and lighting was achieved by the software LabVIEW. The typical control block (\#7 and \#8 channel) was shown in Fig. 3. In For loop, output voltage (4.5V and 4V), which are adjusted by individual LED (or LD) voltage-current characteristics, were supplied with durations determined by a loop counting number (500) and 
sampling rate (500 per second). So LED turns on 1 second and turns off 1 second in this case. As described above, LabVIEW software achieved novel and flexible control of the LED/LDs.

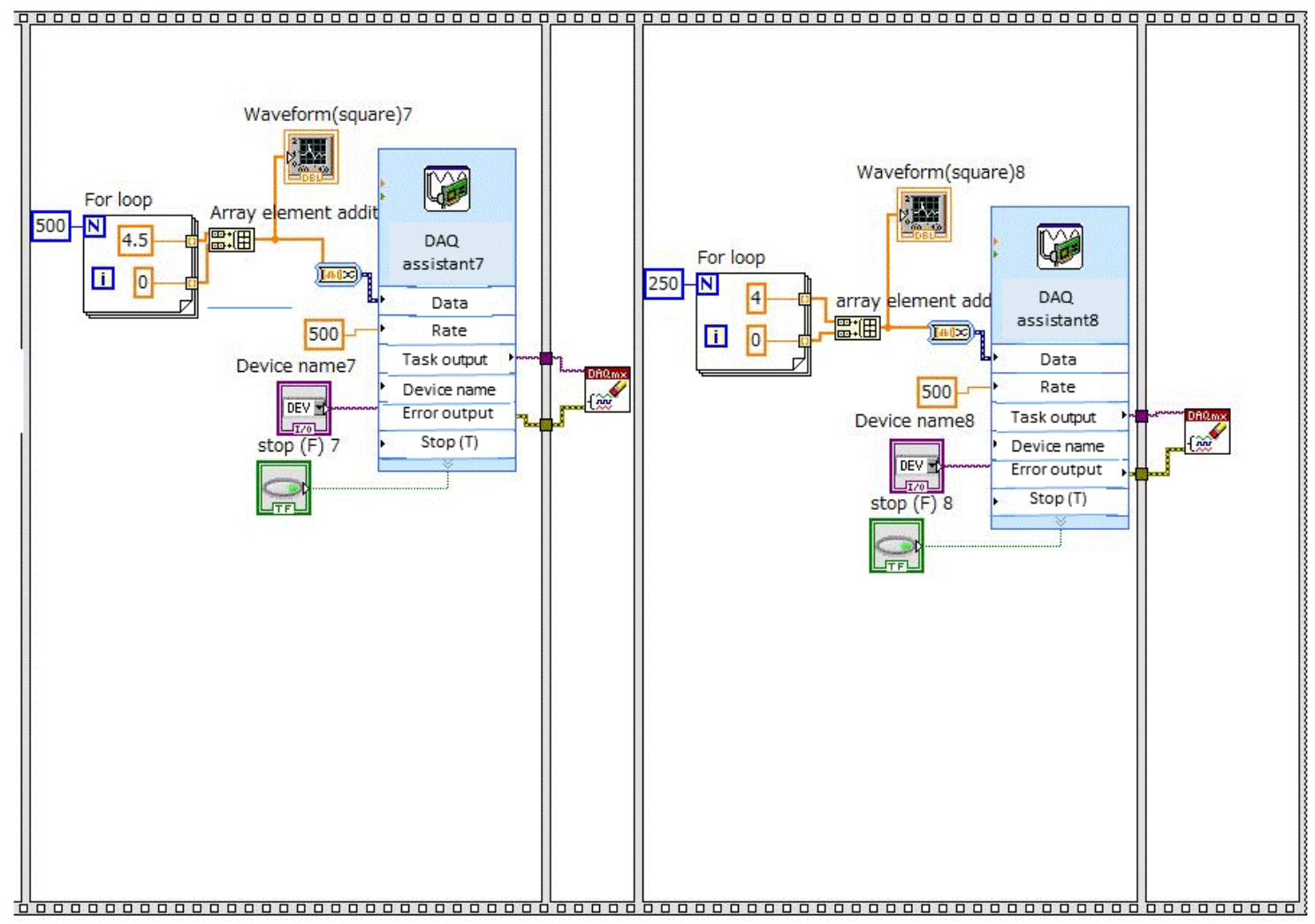

Fig. 3 Typical LabVIEW control block

\section{Thermal images and waveforms}

The specimen used in this measurement was a black-paint coated OHP sheet (polyethylene terephthalate sheet previously used for an over-head projector). A typical temperature waveform obtained for a single channel LED illumination was shown in Fig. 4. In this case, the temperature of an illuminated region increased from 23.5 degree to 24.2 degree. Duration of a temperature waveform (about 1 second) corresponds to an analog output pulse duration 1 second.

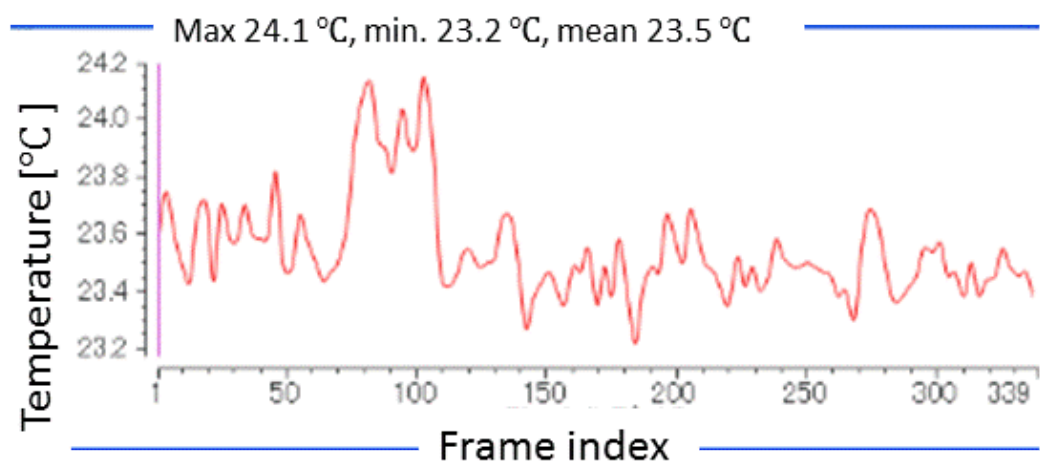

Fig. 4 Temperature waveform for a single channel LED

(LED drive pulse duration 1sec, 30 frames/sec). 
To demonstrate the scanning performance, four lines four LED array combination was used for line-light source scanning experiments. The thermography image after scanning was shown in Fig. 5.

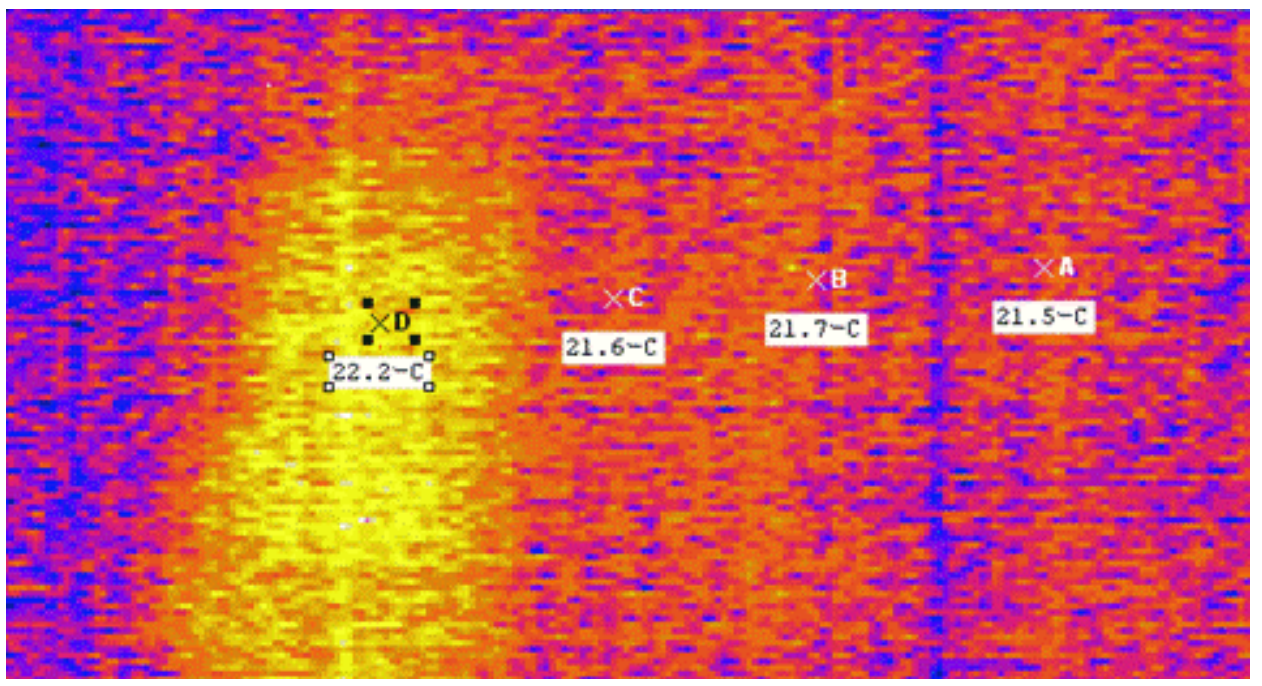

Fig. 5 Thermographic image obtained after scanning by line-LED source from right to left.

(Current is $500 \mathrm{~mA}$ for each $L E D$, scanning speed $4 \mathrm{~mm} / \mathrm{sec}$.)

The temperature waveforms corresponding to the points $A, B, C$, and D separated with distances $8 \mathrm{~mm}$ were shown in Fig. 6. Image sampling rate was 30 frames/sec so that the time separation about 180 frames between temperature peeks at position $\mathrm{A}$ (red) to that of position D (yellow) corresponds to 6 seconds. So that the scanning velocity v is calculated to be

$$
\mathrm{v}=\frac{24 \mathrm{~mm}}{6 \text { seconds }}=4 \mathrm{~mm} / \mathrm{s}
$$

The magnification $\mathrm{m}$ is variable by changing distance between LEDs, lens and specimen so that both the scanning area and scanning speed can be varied easily.

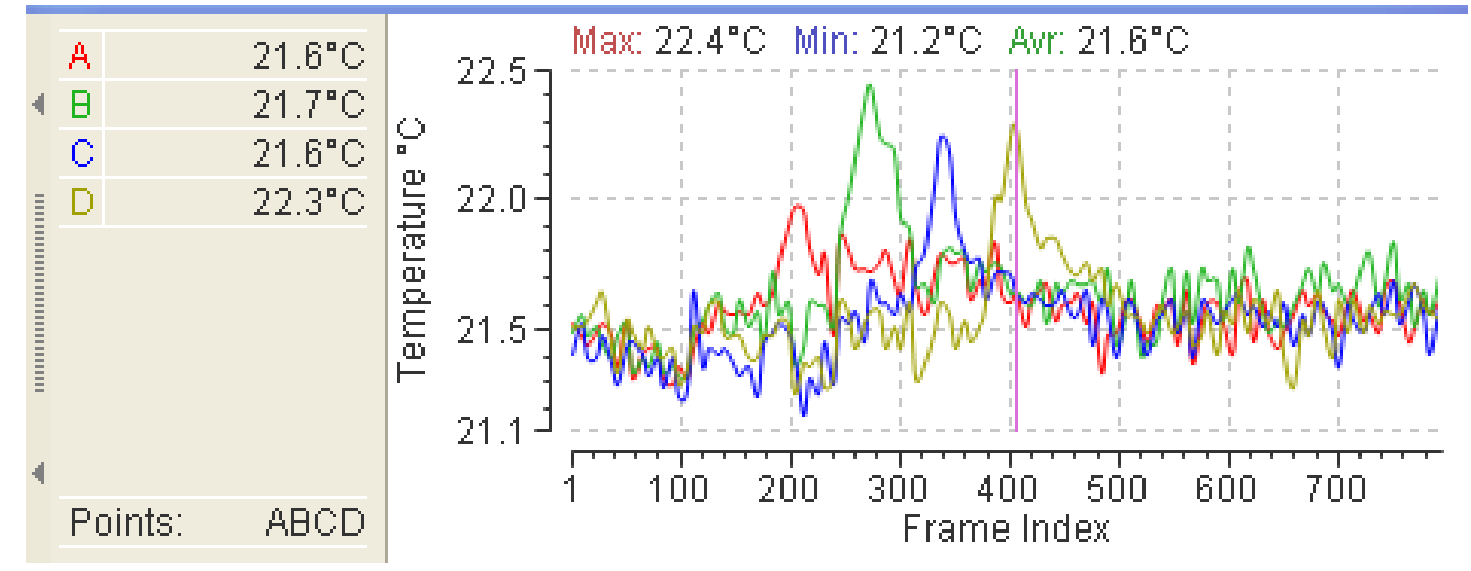

Fig. 6 Temperature waveforms for the points $A$ (red), B (green), $C$ (blue) and D (yellow) shown in Figure 5.

\section{Discussions and conclusion}

In this paper, a whole-electronic scanning line-focus active thermographic imaging system using array of LED/LD was proposed, designed and fabricated for the first time. The LEDs (LDs also available) arranged as an array 
with multi-columns worked as a line-focused light scanner, which is controlled by a software LabVIEW. This system has a capability to work as a variable LED illumination power, variable scanning speed, and the variable duty factor of irradiation. The first two factors play equivalent role to laser-scanner combination adopted in LST. On the other hand, the last factor, or duty factor of illumination, can contribute to decrease constant temperature rise, or baseline shift, shown in the Appendix figure A-1.The present whole electronic LED/LD light scanning scheme is extremely useful in $\mathrm{NDI}$ applications when fabricated in a mobile-sized dimension in the near future. Finally, the authors would like to comment that the application of this scheme to line-emitting light sources (such as line-shaped beam LD/LED) will contribute to decrease component count as $\mathrm{N}$ lines $\mathrm{x} \mathrm{M}$ elements to $\mathrm{N}$ lines and weight saving of the power supply.

\section{REFERENCES}

[1] Cramer, K.E. and W.P. Winfree, - Thermographic Detection and Quantitative Characterization of Corrosion by Application of Thermal Line Source -, Proceedings of SPIE, 3361, pp291-300, 1998.

[2] Hoshimiya T., Hoshimiya J. and Tsuda M., - Line-focus Beam-scan Time-domain Active Thermography with External Control -, Proc., QIRT-2012-186, 2012.

[3] Oswald-Tranta B., Sorger M., - Scanning Pulse Phase Thermography with inductive line source- , Proc., QIRT-2012-264, 2012.

[4] MISTRAS Inc., http:// www.mistrasgroup.com/ products/technologies/thermography.aspx

[5] Wang Y.Q., Kuo P. K., Fabro L. D. and Thomas R. L., - A novel "flying spot" infrared camera for imaging very fast thermal phenomena - Springer Series in Optical Science, Vol. 62, Photoacoustic and Photothermal Phenomena II , 1990.

[6] Gruss C. and Balageas D., -Theoretical and experimental applications of the flying spot camera -, Proc., QIRT 92, 1992.

[7] Hoshimiya T., - Light scanning apparatus and method by electronic scanning -, Japanese patent submission \#2014-2421 (2014.1).

Appendix

Figures A-1 (a)-(d) are temperature waveforms corresponding to the specimens derlin (polyoxymethylene), stainless steel, aluminum and brass, respectively, which were obtained from LST measurements with line-focused DPSS laser [2]. All specimens include simulated inner defects located at the depth of $0.5 \mathrm{~mm}$. Scanning speed was $1 \mathrm{~mm} / \mathrm{sec}$.

In Figures A-2 (a) and (b), waveforms for aluminum specimen obtained with a scanning speed of $1 \mathrm{~mm} / \mathrm{sec}$ and $5 \mathrm{~mm} / \mathrm{sec}$ respectively were shown.

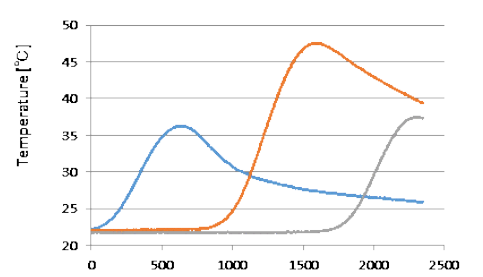

(a) delrin (polyoxymethylene)

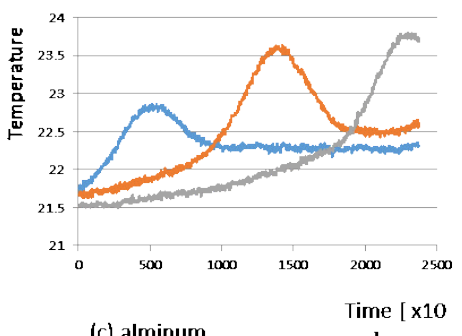

ms]

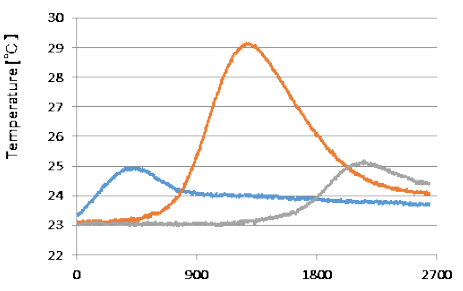

(b) Stainless steel

ms

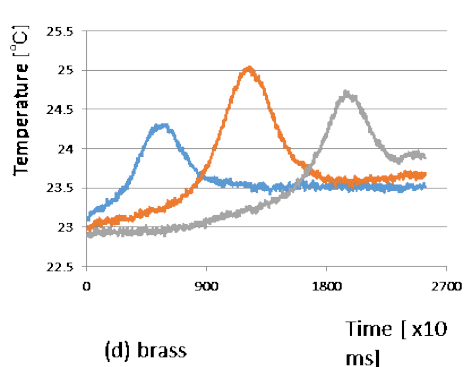

ms]

Fig. A-1 Temperature waveforms of the inner defect for (a)derlin(polyoxymethylene), (b)stainless steel, (c)aluminum and (d)brass specimens observed by the apparatus [2]. 
http://dx.doi.org/10.21611/qirt.2014.222

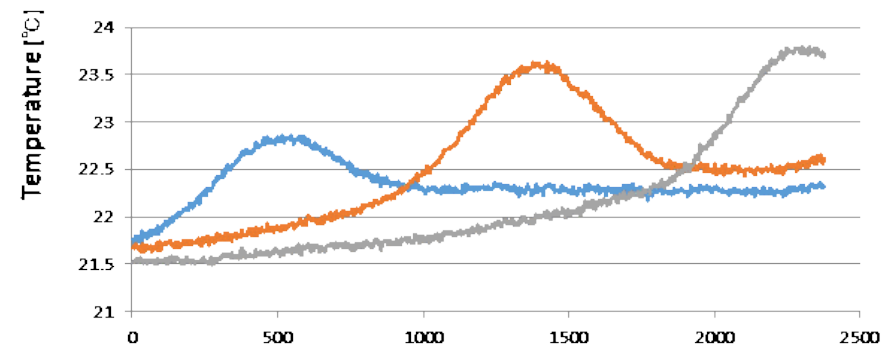

(a) $\mathrm{v}=1 \mathrm{~mm} / \mathrm{sec}$

Time [ $x 10 \mathrm{~ms}]$

before defect

at the defect

after defect

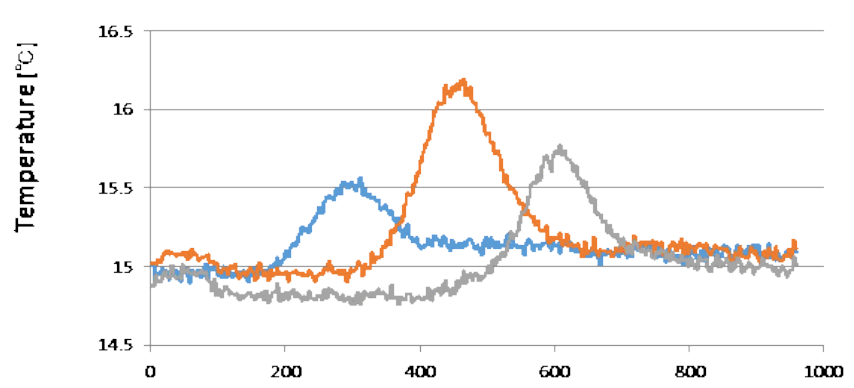

(b) $v=5 \mathrm{~mm} / \mathrm{sec}$

Time [ x10 ms]

Fig. A-2 Temperature waveforms of the inner defect for aluminum specimen obtained with a scanning velocity of (a) $v=1 \mathrm{~mm} / \mathrm{sec}$ and (b) $v=5 \mathrm{~mm} / \mathrm{sec}$. 